\title{
Image Denoising Algorithm Based on Non- Subsampled Contourlet Transform and Bilateral Filtering
}

\author{
C.J. Li, H.X. Yang, Y.Y. Cai, B. Song \\ Physics and optoelectronic \\ Xiangtan University \\ China
}

\begin{abstract}
In order to remove the image noise more effectively, an image denoising algorithm based on Nonsubsampled Contourlet Transform (NSCT) and bilateral filtering in the spatial domain is proposed. The noisy image is first decomposed into multi-scale and multi-directional subbands by NSCT, and direction subbands of each high-pass component is processed by the new threshold function which is obtained by the Bayes threshold that based on stratified noise estimation.. During the reconstruction, the low-pass subband constructed image is further denoisied by the bilateral filtering in the spatial domain. Experimental results demonstrate that the proposed method can improve de-noising performance.
\end{abstract} filtering

Keywords-image de-noising; NSCT; bayes threshold; bilateral

\section{INTRODUCTION}

In the process of collection and transmission, the digital image is often affected by the noise, such as compression, denoising, feature extraction, and inverse problems. So it is necessary to reduce the image noise to improve the quality of the image. Many denoising methods have been developed over the year, there are two main classes of digital image denoising methods: one is processed in the transform domain, the other in a spatial domain. Spatial domain filtering method is to operate directly on the image pixels in the spatial domain. But operating on the image pixel directly will lead to the fuzzy Image edge and details. The bilateral filter [1] and the nonlocal means filter are examples of methods which define the filter kernel in the spatial domain. Tomasi proposed the bilateral filtering (BF) by introducing the weighted function .The bilateral filtering Retains more image details and continuity compared with the traditional spatial filtering method. The transform domain method process the coefficients in the transform domain, there are many transform domain methods, such as Wavelet transform[2], Shearlet transform [3] and Contourlet transform[4]. Multiresolution Bilateral Filtering (MBF) for Image Denoising which is proposed In the literature [5] combine two popular filters for the two domains, An input image is decomposed into its detail and approximation subbands through wavelet decomposition, bilateral filtering is applied to the approximation subbands, at the same time wavelet thresholding is applied to the detail subbands, But the two-dimensional wavelet transform can only extract information in two directions. Nonsubsampled Contourlet Transform (NSCT) [6] is a multi-directional and multi-scale decomposition. We propose a method based on Non-subsampled Contourlet Transform and Bilateral Filtering. The new Threshold function is used in the detail subbands.

\section{ALGORITHM}

\section{A. Nonsubsampled Contourlet Transform}

Wavelet transform is widely used in many signal processing and communication applications for its timefrequency localization and multi-scale and multi-resolution. Owing to the geometric information, the nonsubsampled contourlet transform achieves better results than wavelet transform in many image analysis applications, such as denoising. We introduce the construction of the NSCT as follow: For the filter design, we refer readers to [4]. To achieve the shift-invariance, the NSCT employs nonsubsampled pyramids for multiscale decomposition, and nonsubsampled directional filter banks (DFB) for directional decomposition. First, a nonsubsampled pyramid split the input into a lowpass subband and a highpass subband. Then a nonsubsampled DFB decomposes the highpass subband into several directional subbands. The scheme is iterated repeatedly on the lowpass subband.

\section{B. Bayesian Denoiser}

The goal of image denoising is to estimate the original image $x$ from a noise-contaminated image $y=x+\beta$ with a stationary variance $\sigma_{\beta}{ }^{2}=\operatorname{Var}[\beta]$. The noisy image is decomposed to the lowpass subband and the highpass suband, The noise variance of different directional subband is no longer equal to the image noise variance in the transform domain[7], we adopt the method of hierarchical estimation to obtain the noise standard deviation in order to reflect the noise standard deviation under different scales[8], eqn. (1) shows the threshold in the Bayesian framework.

$$
t=\frac{\sigma_{n}^{2}(j, k)}{\sigma_{x}(j, k)}
$$

$\sigma_{n}(j, k)$ is the noise variance and $\sigma_{x}(j, k)$ is the standard deviation in the formula, $j, k$ represents the scale and the direction. We, use eqn. (2) to estimate the noise standard deviation in NSCT domain.

$$
\sigma_{\beta}(j, k)=\frac{\text { median }(|Y(l, k)|)}{0.6745}
$$


Where the $Y(l, k)$ is all of the high frequency coefficients in the direction $k$ and in the $j$ layer after the nonsubsampled contourlet transform decomposition .We estimate the noise variance of the subband coefficients in the different scales and directions according to eqn. (3).

$$
\sigma_{y}^{2}(j, k)=\frac{1}{L W} \sum_{l=1}^{L} \sum_{w=1}^{W} c_{l, w}^{2}(j, k)
$$

The parameters $L$ and $W$ are the length and width of the image, $C_{l, w}(j, k)$ is the coefficients of the NSCT. To estimate $\sigma_{x}(j, k)$ according to eqn.(4), the noisy image standard deviation of subband coefficients.

$$
\sigma_{x}^{2}(j, k)=\max \left(\sigma_{y}^{2}(j, k)-\sigma_{n}^{2}(j, k), 0\right)
$$

\section{The Bilateral Filter}

The bilateral filter is the nonlinear filter based on the summary of the thinking of Overt on et al. [9] and the basic of the gaussian filtering algorithm. The filter takes a weighted sum of the pixels in a local neighborhood; the weights depend on both the intensity distance and the spatial distance [10]. Edges are preserved well while noise is averaged out. at a pixel location $g(p, q)$, the output $f(p, q)$ of the bilateral filter is calculated as follows:

$$
f(p, q)=\frac{\sum_{(m, m) \in S_{p, q}} \omega(m, n) \times g(m, n)}{\sum_{(m, n) \in S_{p, q}} \omega(m, n)}
$$

Where $S_{p, q}$ represents the spatial neighborhood of the center $g(p, q)$, the size of the neighborhood is $(2 h+1) \times(2 h+1)$, the radius of the spatial neighborhood is $h$. the intensity distance and the spatial distance as follow:

$$
\omega(m, n)=\omega_{\delta}(m, n) \omega_{\gamma}(m, n)=e^{\frac{|m-p|+|n-q|}{2 \sigma_{\delta}^{2}}} \times e^{\frac{(m-p)+(n-q)}{2 \sigma_{\gamma}^{2}}}
$$

Where $\sigma_{\delta}$ and $\sigma_{r}$ are parameters controlling the fall-off of the weights in spatial and intensity domains respectively. When $h$ increases gradually, the smoother the image at the same time. Although the bilateral filter is being used more and more widely, there is not much theoretical basis on selecting the optimal $h$. According to the noise standard deviation $\sigma_{\beta}$, we propose a adaptive function for parameter $h$ as follow:

$$
h=\left\{\begin{array}{cc}
1, & \mu \leq 1 \\
\text { round }\left[\sigma_{\beta}+\left(10 e^{\sigma_{\beta}}-1\right)^{2}+10 e^{\sigma_{\beta}}\right] & \mu>1
\end{array}\right.
$$

Where the variable $\mu$ is

$$
\mu=10\left(e^{\sigma_{\beta}}-1\right)
$$

When the noise standard deviation $\sigma_{\beta}$ increase, the variable $\mu$ will be increase, and the filter radius will be increase gradually.

\section{The Improved Threshold Function}

We decompose an noisy image into low-frequency and high-frequency components by NSCT, and apply NSCT thresholding on the detail subbands. The commonly used threshold functions are soft-thresholding and hardthresholding function [11].

Hard-thresholding function:

$$
C_{j, k}^{\prime}=\left\{\begin{array}{cc}
C_{j, k}, & \left|C_{j, k}\right| \geq t \\
0, & \left|C_{j, k}\right|<t
\end{array}\right.
$$

Soft-thresholding function:

$$
C_{j, k}^{\prime}=\left\{\begin{array}{cl}
\operatorname{sgn}\left(\left|C_{j, k}\right|\right)\left(\left|C_{j, k}\right|-t\right), & \left|C_{j, k}\right| \geq t \\
0, & \left|C_{j, k}\right|<t
\end{array}\right.
$$

Where $C_{j, k}$ is the NSCT coefficients and $t$ is the threshold, $C^{\prime}{ }_{j, k}$ is the processed coefficients. Hard-threshold function can effectively preserve the image edge and local feature, but the noisy image denoised by hard-thresholding function will cause the ringing and the pseudo Gibbs phenomenon [12]. Softthresholding function is continuous, It overcomes the shortcomings of the discontinuity of hard threshold, so the signal after denoising become more smooth, but it will cause the edge and local feature lose, because the soft-thresholding function cut the coefficients with larger absolute value, which affect the reconstructed image and the approximation degree of the original image. This paper proposed a new thresholding function, the improved threshold function makes up for deficiencies of each threshold functions better.

$$
C_{j, k}^{\prime}=\left\{\begin{array}{cc}
C_{j, k}, & \left|C_{j, k}\right|>\lambda t \\
\operatorname{sgn}\left(C_{j, k}\right) \frac{\lambda\left(\left|C_{j, k}\right|-t\right)}{\lambda-1} e^{\left(C_{j, k}-\lambda t\right)}, t \leq & \left(\left|C_{j, k}\right|-t\right) \leq \lambda t \\
0, & \left|C_{j, k}\right|<t
\end{array}\right.
$$

Where $t$ is the Bayes thresholding and $\lambda$ is a parameter, the proposed thresholding function not only keep the edge feature of the hard-threshold, but also consider the continuity of the soft- thresholding. It can reduce the phenomenon of ringing, pseudo Gibbs and the unclear edge.

We can prove the continuity of the proposed thresholding function: when the coefficient is $\left|C_{j, k}\right|>\lambda t$ and approaching to $\lambda t$, the threshold approach to $C_{j, k}$, when the coefficient in the condition of $t \leqslant\left(\left|C_{j, k}\right|\right) \leqslant \lambda t$ and approaching to $\lambda t$, the threshold approach to $C_{j, k}$ too, In the same way, when the coefficient is approaching to $t$, the threshold approach to 0 , so the proposed thresholding function is continuous.

\section{E. Image Denoising Algorithm}

In this paper, we get the high and the low frequency decomposition coefficients from the input image by the NSCT. The high frequency coefficients is de-noised based on stratified noise estimation and adaptive Bayes threshold in the NSCT domain, meanwhile, the low frequency reconstruction image is processed by the bilateral filter.

We summarize our method in the following algorithm:

1) Compute the input noisy image for a low-subband and several high-subbands through the NSCT decomposition 
2) Estimate the Bayesian threshold of the input detail subbands at each decomposition level according to eqn.(1) and use the new threshold function according to eqn.(11), reset the low -frequency coefficient of to null array. We gain the highfrequency reconstructed subband image by reconstructing the enhanced image from the modified NSCT coefficients.

3) Reconstruct the low-frequency subband image from the NSCT coefficients has been reset the detail subband coefficients, and apply bilateral filtering on the low-frequency subband image in the image domain.

4) The denoising image can be obtained by adding the lowfrequency subband image and the high-frequency subband image.

\section{THE RESULT AND DISCUSSION}

We have conducted some experiments to compare the performance of the proposed algorithm quantitatively and visually. To do a quantitative comparison, we simulated noisy images by adding white Gaussian noise with standard deviations 10,20, and 30. These noisy images were then denoised by using the above algorithms and the PSNR results were calculated. we use the standard grayscale Lena and Barbara as visual comparisons. The pixels of the text grayscale image is $512 \times 512$, For each standard test image. PSNR results for three methods are included in Table I. BF is the bilateral filter, MBF represent the method proposed in the article [3], the third method is the proposed method. For the proposed method, we set the parameters as follow: $\left[\sigma_{\delta}\right.$, $\left.\sigma_{r}\right]=[4,0.23], \lambda=2.0$ turned out to be a better choice in terms of Signal-to-Noise Ratio(PSNR), For the NSCT, we decompose the image into three levels, and set the direction respectively as follow:[2,4,8]. we choice the "maxflat" as nonsubsampled pyramids and "dmaxflat7" as nonsubsampled directional filter banks in Matlab version 7.0.

TABLE I. PSNR (DB) COMPARISON IN DIFFERENT NOISE STANDARD DEVIATION.

\begin{tabular}{|c|c|c|c|c|c|c|}
\hline & \multicolumn{3}{|c|}{ Lena } & \multicolumn{3}{c|}{ Boat } \\
\hline$\sigma_{n}$ & 10 & 20 & 30 & 10 & 20 & 30 \\
\hline BF & 33.60 & 30.33 & 28.54 & 32.02 & 28.40 & 26.57 \\
\hline MBF & 34.48 & 31.28 & 29.33 & 32.58 & 29.25 & 27.24 \\
\hline NBF & 34.70 & 31.81 & 29.55 & 33.03 & 29.55 & 27.24 \\
\hline & \multicolumn{3}{|c|}{ Barbara } & \multicolumn{3}{c|}{ House } \\
\hline$\sigma_{n}$ & 10 & 20 & 30 & 10 & 20 & 30 \\
\hline BF & 31.37 & 27.02 & 24.69 & 33.77 & 29.63 & 28.11 \\
\hline MBF & 31.79 & 27.74 & 25.61 & 34.62 & 31.37 & 29.24 \\
\hline NBF & 31.99 & 29.12 & 26.37 & 38.40 & 34.50 & 32.62 \\
\hline
\end{tabular}

As seen in the PSNR results of Table1, the proposed method is $1.2 \mathrm{~dB}$ better than the bilateral filter and $2.0 \mathrm{~dB}$ better than MBF on average. PSNR comparisons with simulated white Gaussian noising is only a part of the evaluation standard, another common standard is the real performance of image denoising algorithms. Fig.1.is the Lena denoising result, the Fig.1.shows the comparison of NBF and other algorithm in the condition of $\sigma_{n}=20$, the experiments with test image demonstrate effectiveness of the proposed method.
Fig.1. and Fig.2. shows the test image "Lena" and "Barbara", we compare the bilateral filter, the MBF method and the proposed method NBF in Fig(c), (d) and(e) respectively. The shoulder of the Lena in Fig (e) is smoother than the bilateral filter in the Fig (c), the detail of the trousers in Fig (e) is clearer than the MBF in the Fig (d).
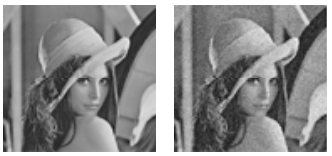

(a) Original (b) Noisy Image

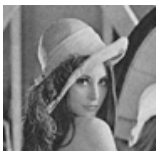

(c)BF

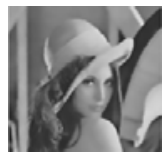

(d)MBF

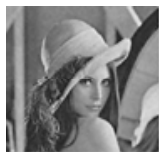

(e)NBF
FIGURE I. COMPARISON OF NBF AND OTHER ALGORITHM IN THE CONDITION OF $\Sigma \mathrm{N}=20$.
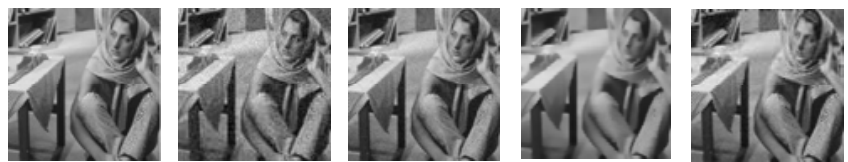

(a) Original (b) Noisy Imag

(c)BF (d)MBF

(e)NBF

FIGURE II. COMPARISON OF NBF AND OTHER ALGORITHM IN THE CONDITION OF $\Sigma \mathrm{N}=20$.

\section{CONCLUSIONS}

In this paper we present an image denoising algorithm which integrates the nonsubsampled contourlet transform thresholding filtering and the bilateral filter, we decompose a digital image into high-frequency and low-frequency components, and apply nonsubsampled contourlet transform thresholding on the detail subbands and bilateral filtering on the approximation subbands. The experiments with data demonstrate the effectiveness of the proposed method. The new threshold function can remove the image noise more effectively and achieve higher PSNR.

\section{REFERENCES}

[1] Tomasi C., Manduchi R., Bilateral filtering for gray and color images. In Proceeding of the 1998 IEEE International Conference on Computer Vision, Bombay, India, pp. 839-846, 1998

[2] Weyrich N., Warhola G. T., Wavelet shrinkage and generalized cross validation for image denoising. IEEE Transactions on Image Proceeding, 7 (1), pp. 82-90, 1998.

[3] Do M N, Vetterli M.,The Contourlet Transform: An Efficient Directional Multireslution Image Representation. IEEE Trans. on Image Processing, 14(12), pp. 2091-2106, 2005

[4] Guo K., Labate D., Optimally Sparse Multidimensional Representation using Shearlets. SIAM J Math Anal, 39( 1), pp. 298-318, 2007.

[5] M. Zhang, Gunturk B. K., Multi-resolution bilateral filtering for image denoising. IEEE Trans on Image Processing, 17(12), pp. 2324-2333, 2008.

[6] Cunha A L, J.P. Zhou, Do M.N., The Non-subsampled Contourlet: Theory. Design and Applications. IEEE Trans. on Image Processing, 15(10), pp. 1779-1793, 2006

[7] L. Ding, L.J. Wang, Z.H. Jia, Adaptive Image Denoising Algorithm Integrating Bayes with Contourlet Transform. Laser Journal, 34(6), pp. 43-45, 2013

[8] L. Li, H.X. Yang, Adaptive Threshold De-noising Algorithm Combined with Fourier Transform and Non-subsampled Contourlet Transform. Computer Engineering, 38(17), pp. 238-241, 2012.

[9] Z.Q. Zhang, W.Y. Wang, A Modified Bilateral Filtering Algorithm. Journal of Image and Graphics, 14(3), pp. 443-447, 2009. 
[10] N.N. Li, J.J. Cao, B. Li et al. Reweighted Global Bilateral Filtering Based on Normal Regularization. Journal of Computer-Aided Design \& Computer Graphics , 26(3), pp. 370-377, 2014.

[11] F. Tang, H.X. Yang, Y.W. Zeng, et al, Image denosing algorithm based on improved Contourlet transform. Computer Engineering and Applications, 50(5), pp. 132-136, 2014.

[12] Y. Yang, W.J. Zheng, S.Y. Huang, et al, Multi-focus image fusion based on human visual perception characteristic in Non-subsampled Contourlet transform domain. Journal of Image and graphics, 19(3), pp. 447-455, 2014. 